\title{
Analytical analysis of the Pennes bioheat transfer equation with sinusoidal heat flux condition on skin surface
}

\author{
Tzu-Ching Shih ${ }^{\mathrm{a}}$, Ping Yuan ${ }^{\mathrm{b}}$, Win-Li Lin ${ }^{\mathrm{c}, \mathrm{d}}$, Hong-Sen Kou ${ }^{\mathrm{e}, *}$ \\ ${ }^{a}$ Department of Medical Radiology Technology, China Medical University, Taiwan \\ ${ }^{\mathrm{b}}$ Department of Mechanical Engineering, Lee-Ming Institute of Technology, Taiwan \\ ${ }^{\mathrm{c}}$ Institute of Biomedical Engineering, National Taiwan University, Taiwan \\ d Medical Engineering Research Division, National Health Research Institutes, Taiwan \\ ${ }^{\mathrm{e}}$ Department of Mechanical Engineering, Tatung University, Taiwan
}

Received 20 February 2006; received in revised form 13 October 2006; accepted 16 October 2006

\begin{abstract}
This study focuses on the effect of the temperature response of a semi-infinite biological tissue due to a sinusoidal heat flux at the skin. The Pennes bioheat transfer equation such as $\rho_{\mathrm{t}} c_{\mathrm{t}}(\partial T / \partial t)+W_{\mathrm{b}} c_{\mathrm{b}}\left(T-T_{\mathrm{a}}\right)=k \partial^{2} T / \partial x^{2}$ with the oscillatory heat flux boundary condition such as $q(0, t)=q_{0} \mathrm{e}^{\mathrm{i} \omega t}$ was investigated. By using the Laplace transform, the analytical solution of the Pennes bioheat transfer equation with surface sinusoidal heating condition is found. This analytical expression is suitable for describing the transient temperature response of tissue for the whole time domain from the starting periodic oscillation to the final steady periodic oscillation. The results show that the temperature oscillation due to the sinusoidal heating on the skin surface is unstable in the initial period. Further, it is unavailable to predict the blood perfusion rate via the phase shifting between the surface heat flux and the surface temperature. Moreover, the lower frequency of sinusoidal heat flux on the skin surface induces a more sensitive phase shift response to the blood perfusion rate change, but extends the beginning time of sampling because of the avoidance of the unavailable first cyclic oscillation.
\end{abstract}

() 2006 IPEM. Published by Elsevier Ltd. All rights reserved.

Keywords: Bioheat transfer equation; Sinusoidal heat flux; Analytical solution

\section{Introduction}

The temperature response and blood perfusion during surface heating have been studied by several investigators [1-8]. In these medical problems, heat transfer analysis often needs to simultaneously deal with transient and spatial heating both on the skin surface and inside biological bodies. The Pennes bioheat transfer equation, which describes the exchange magnitude of heat transfer between tissue and blood, is widely used to solve the temperature distribution for thermal therapy [3-8]. Also, the Pennes bioheat transfer equation is based on the assumption that all heat transfer between the tissue and the blood occurs in the capillaries. In other words, this continuum approach neglects the local effects where

\footnotetext{
* Corresponding author. Tel.: +886228728218.

E-mail address: hskou@ttu.edu.tw (H.-S. Kou).
}

"thermally significant" blood vessels do not appear in the temperature field. Moreover, Shih et al. [9] performed an investigation where the cooling effects of thermally significant blood vessels (larger than $0.2 \mathrm{~mm}$ in diameter) were demonstrated in detail. Kou et al. [10] also solved the analytical solution of a transient bioheat transfer equation based on the porous medium property, which describes the encompassing directional effect of blood flow. For localized hyperthermia, Ocheltree and Frizzell [11,12] demonstrated the analytical formulations for both transient and steady state of three tumor models via the Pennes bioheat transfer equation.

For a sinusoidal heating on the skin surface problem, Liu and $\mathrm{Xu}$ [13] performed a closed-form analytical solution of the Pennes bioheat transfer equation. Their analytical solution is suitable for the long run steady periodic temperature oscillation. However, their analytical solution cannot satisfy 
the starting transient temperature response. For the analysis with various boundary conditions on the skin surface, Liu and $\mathrm{Xu}$ [13] also found that a phase shift between the surface temperature and the heat flux can be utilized to estimate the blood perfusion in living tissues. Using the Monte Carlo algorithm, Deng and Liu [2] also presented accurate correlations between skin thermal image information and human pathophysiology for possible diagnostics. Moreover, Deng and Liu [14] investigated several analytical solutions to the bioheat transfer equation problems with space or transient heating on skin surface or inside bodies obtained by the Green's function method. Ng and Chua [6,8] numerically solved the Pennes bioheat transfer equation using the finite-difference (1D) and the finite-element (2D) analysis for predicting the state of skin burns. Furthermore, $\mathrm{Ng}$ and Sudharsan [15,16] applied the four-noded tetrahedrons and a mesh less-sensitive numerical approximation to model the surface temperature and tissue temperature of a normal breast and a breast with a tumor in order to develop an expert system for breast cancer detection. They also found that the surface temperature distribution changes with the size and depth of the tumor. In addition, there are some studies on the heating kinetics obtained during millimeter wave exposures of human skin for a short period of time [17-20]. Thus, the transient response of temperature is very important for estimation of the blood perfusion in thermal treatment. Therefore, the primary aim of this study is to derive the exact solution of the Pennes bioheat transfer equation prescribed with the sinusoidal heating boundary condition.

\section{Methods}

The analysis of the transient temperature field in the domain of blood perfused tissues subjected to oscillated heating condition is presented. Because of the geometrical features of the skin, the heat exchange in domain considered is assumed to be one-dimensional. In addition, this study considers an oscillated boundary condition such as $q(0, t)=q_{0} \mathrm{e}^{\mathrm{i} \omega t}$ to explore the temperature variation of the tissues.

For the transient problem, the one-dimensional Pennes bioheat transfer equation is given as:

$\rho_{\mathrm{t}} c_{\mathrm{t}} \frac{\partial T}{\partial t}+W_{\mathrm{b}} c_{\mathrm{b}}\left(T-T_{\mathrm{a}}\right)=k \frac{\partial^{2} T}{\partial x^{2}}$

where $\rho_{\mathrm{t}}$ is tissue density, $c_{\mathrm{t}}$ the specific heat of tissue, $T$ the tissue temperature, $t$ the time, $W_{\mathrm{b}}$ the blood perfusion rate, $c_{\mathrm{b}}$ the specific heat of blood, $T_{\mathrm{a}}$ the supplying arterial blood temperature, $k$ the thermal conductivity of tissue, and $x$ is the distance from the skin surface.

The oscillatory heat flux boundary condition is described as follows:

$$
-\left.k \frac{\partial T}{\partial x}\right|_{x=0}=q_{0} \mathrm{e}^{\mathrm{i} \omega t}
$$

where $q_{0}$ is heat flux on the skin surface, and $\omega$ is heating frequency.

For simplicity, the dimensionless variables are defined as:

$$
\begin{aligned}
z & \equiv \omega t, \quad \alpha \equiv \frac{k}{\rho_{\mathrm{t}} c_{\mathrm{t}}}, \quad X \equiv \sqrt{\frac{\omega}{\alpha}} x, \quad c_{1} \equiv \frac{W_{\mathrm{b}} c_{\mathrm{b}}}{\rho_{\mathrm{t}} c_{\mathrm{t}} \omega}, \\
\theta & \equiv \frac{k\left(T-T_{\mathrm{a}}\right)}{q_{0}} \sqrt{\frac{\omega}{\alpha}}
\end{aligned}
$$

Eq. (1) can be rewritten in terms of dimensionless variables and becomes:

$\frac{\partial \theta}{\partial z}+c_{1} \theta=\frac{\partial^{2} \theta}{\partial X^{2}}$

Alternatively, the dimensionless boundary condition is expressed as:

$\left.\frac{\partial \theta}{\partial X}\right|_{X=0}=-\mathrm{e}^{\mathrm{i} z}$

Taking the Laplace transform of Eq. (4), then it becomes:

$\frac{\mathrm{d}^{2} \Phi}{\mathrm{d} X^{2}}-\left(s+c_{1}\right) \Phi=0$

The general solution of Eq. (6) can be obtained as:

$\Phi=c_{2} \mathrm{e}^{-\sqrt{s+c_{1}} X}$

Based on Eq. (7), the dimensionless boundary condition can be rewritten as:

$\left.\frac{\mathrm{d} \Phi}{\mathrm{d} X}\right|_{X=0}=-\sqrt{s+c_{1}} c_{2}$

Taking the Laplace transform of Eq. (5), it becomes:

$\left.\frac{\mathrm{d} \Phi}{\mathrm{d} X}\right|_{X=0}=-\frac{1}{s-\mathrm{i}}$

Hence, the coefficient $c_{2}$ gives:

$c_{2}=\frac{1}{(s-\mathrm{i}) \sqrt{s+c_{1}}}$

Substituting Eq. (10) into Eq. (7), and it is as follows:

$\Phi=\frac{\mathrm{e}^{-\sqrt{s+c_{1}} X}}{(s-\mathrm{i}) \sqrt{s+c_{1}}}$

Taking the Inverse Laplace transform of Eq. (11), it obtains:

$$
\begin{aligned}
\theta= & \frac{\mathrm{e}^{\mathrm{i} z}}{2 \sqrt{c_{1}+i}}\left\{\mathrm{e}^{-\sqrt{c_{1}+\mathrm{i} X}} \operatorname{erfc}\left[\frac{X}{2 \sqrt{z}}-\sqrt{\left(c_{1}+\mathrm{i}\right) z}\right]\right. \\
& \left.-\mathrm{e}^{\sqrt{c_{1}+\mathrm{i}} X} \operatorname{erfc}\left[\frac{X}{2 \sqrt{z}}+\sqrt{\left(c_{1}+\mathrm{i}\right) z}\right]\right\}
\end{aligned}
$$

Based on Eq. (12), the temperature response on the skin surface becomes:

$\left.\theta\right|_{X=0}=\frac{\mathrm{e}^{\mathrm{i} z}}{\sqrt{c_{1}+\mathrm{i}}} \operatorname{erf}\left[\sqrt{\left(c_{1}+\mathrm{i}\right) z}\right]$ 
Combining Eq. (5) with Eq. (13), the relationship between surface heat flux oscillation and surface temperature response can be found as:

$\left.\theta\right|_{X=0}=-\left.\frac{\operatorname{erf}\left[\sqrt{\left(c_{1}+\mathrm{i}\right) z}\right]}{\sqrt{c_{1}+\mathrm{i}}} \frac{\partial \theta}{\partial X}\right|_{X=0}$

Finally, the solution of one-dimensional transient bioheat transfer equation with the periodic heating at the surface of living tissue is given by:

$$
\begin{aligned}
T= & T_{\mathrm{a}}+\frac{q_{0}}{2 k}\left(\sqrt{\frac{\alpha}{\left(c_{1}+\mathrm{i}\right) \omega}} \mathrm{e}^{\mathrm{i} \omega t}\right)\left\{\mathrm{e}^{-\sqrt{\frac{\left(c_{1}+\mathrm{i}\right) \omega}{\alpha}} x} \operatorname{erfc}\right. \\
& \times\left[\frac{x}{\sqrt{4 \alpha t}}-\sqrt{\left(c_{1}+\mathrm{i}\right) \omega t}\right] \\
& \left.-\mathrm{e} \sqrt{\frac{\left(c_{1}+\mathrm{i}\right) \omega}{\alpha}} x \operatorname{erfc}\left[\frac{x}{\sqrt{4 \alpha t}}+\sqrt{\left(c_{1}+\mathrm{i}\right) \omega t}\right]\right\}
\end{aligned}
$$

Introducing the parameter $\beta=\sqrt{\left(W_{\mathrm{b}} c_{\mathrm{b}} / k\right)+(\mathrm{i} \omega / \alpha)}$, Eq. (15) can be rewritten as:

$$
\begin{aligned}
T= & T_{\mathrm{a}}+\frac{q_{0}}{2 k \beta} \mathrm{e}^{\mathrm{i} \omega t}\left[\mathrm{e}^{-\beta x} \operatorname{erfc}\left(\frac{x}{\sqrt{4 \alpha t}}-\beta \sqrt{\alpha t}\right)-\mathrm{e}^{\beta x} \mathrm{erfc}\right. \\
& \left.\times\left(\frac{x}{\sqrt{4 \alpha t}}+\beta \sqrt{\alpha t}\right)\right]
\end{aligned}
$$

Based on Eq. (16), the two temporal conditions can be derived as:

$\left.T\right|_{t \rightarrow 0}=T_{\mathrm{a}}$

$\left.T\right|_{t \rightarrow \text { larger time }}=T_{\mathrm{a}}+\frac{q_{0}}{k \beta} \mathrm{e}^{\mathrm{i} \omega t} \mathrm{e}^{-\beta x}$

The above equations can be used to predict the transient temperature profiles in living tissue subjected by a sinusoidal heating at the skin surface. For the fixed position, Eq. (18) indicates that the temperature variation will approach its final steady periodic oscillation after a longer time duration.

In addition to the above general solutions, the three different but simple heating situations are also demonstrated in the following cases.

Special case $\mathrm{I}: W_{\mathrm{b}} c_{\mathrm{b}}=0$ (i.e., no blood perfusion term)

The solution of this case can be directly obtained from Eq. (15) by assuming $c_{1}=0$.

$$
\begin{aligned}
T= & T_{\mathrm{a}}+\frac{q_{0}}{2 k}\left(\sqrt{\frac{\alpha}{\mathrm{i} \omega}} \mathrm{e}^{\mathrm{i} \omega t}\right)\left\{\mathrm{e}^{-\sqrt{\frac{\mathrm{i} \omega}{\alpha}} x} \operatorname{erfc}\right. \\
& \left.\times\left[\frac{x}{\sqrt{4 \alpha t}}-\sqrt{\mathrm{i} \omega t}\right]-\mathrm{e}^{\sqrt{\frac{\mathrm{i} \omega}{\alpha}} x} \operatorname{erfc}\left[\frac{x}{\sqrt{4 \alpha t}}+\sqrt{\mathrm{i} \omega t}\right]\right\}
\end{aligned}
$$

Special case II: $q(0, t)=q_{0}$ (i.e., constant heat flux)
In this special case, the governing equation is the same with Eq. (1) and the boundary condition is given by:

$-\left.k \frac{\partial T}{\partial x}\right|_{x=0}=q_{0}$

The dimensionless variables are assumed as follows:

$X^{\prime} \equiv x \sqrt{\frac{W_{\mathrm{b}} c_{\mathrm{b}}}{k}}, \quad \alpha \equiv \frac{k}{\rho_{\mathrm{t}} c_{\mathrm{t}}}, \quad z^{\prime} \equiv \frac{W_{\mathrm{b}} c_{\mathrm{b}}}{\rho_{\mathrm{t}} c_{\mathrm{t}}} t$,

$\theta^{\prime} \equiv \frac{T-T_{\mathrm{a}}}{q_{0}} \sqrt{k W_{\mathrm{b}} c_{\mathrm{b}}}$

Eq. (1) can be rewritten in a simple form as:

$\frac{\partial \theta^{\prime}}{\partial z^{\prime}}+\theta^{\prime}=\frac{\partial^{2} \theta^{\prime}}{\partial X^{\prime 2}}$

The dimensionless form of Eq. (20) can be expressed as:

$$
\left.\frac{\partial \theta^{\prime}}{\partial X^{\prime}}\right|_{X^{\prime}=0}=-1
$$

Taking the Laplace transform of Eqs. (22) and (23), then they become:

$\frac{\mathrm{d}^{2} \Theta}{\mathrm{d} X^{2}}-(s+1) \Theta=0$

and

$\left.\frac{\mathrm{d} \Theta}{\mathrm{d} X^{\prime}}\right|_{X^{\prime}=0}=-\frac{1}{s}$

The solution of Eqs. (24) and (25) is:

$\Theta=\frac{\mathrm{e}^{-\sqrt{s+1} X^{\prime}}}{s \sqrt{s+1}}$

Using the Inverse Laplace transform, the dimensionless solution can be written as:

$$
\begin{aligned}
\theta^{\prime}= & \frac{1}{2}\left[\mathrm{e}^{-X^{\prime}} \operatorname{erfc}\left(\frac{X^{\prime}}{2 \sqrt{z^{\prime}}}-\sqrt{z^{\prime}}\right)-\mathrm{e}^{X^{\prime}} \operatorname{erfc}\right. \\
& \left.\times\left(\frac{X^{\prime}}{2 \sqrt{z^{\prime}}}+\sqrt{z^{\prime}}\right)\right]
\end{aligned}
$$

Using the Eq. (21) for the expression of primitive variables given above, Eq. (27) becomes:

$$
\begin{aligned}
T= & T_{\mathrm{a}}+\frac{q_{0}}{\sqrt{4 k W_{\mathrm{b} c_{\mathrm{b}}}}}\left[\mathrm{e}^{-\sqrt{\frac{W_{\mathrm{b}} c_{\mathrm{b}}}{k}} x} \operatorname{erfc}\right. \\
& \times\left(\frac{x}{\sqrt{4 \alpha t}}-\sqrt{\frac{W_{\mathrm{b}} c_{\mathrm{b}}}{\rho_{\mathrm{t}} c_{\mathrm{t}}} t}\right) \\
& \left.-\mathrm{e} \sqrt{\frac{W_{\mathrm{b}} c_{\mathrm{b}}}{k}} x \operatorname{erfc}\left(\frac{x}{\sqrt{4 \alpha t}}+\sqrt{\frac{W_{\mathrm{b}} c_{\mathrm{b}}}{\rho_{\mathrm{t}} c_{\mathrm{t}}} t}\right)\right]
\end{aligned}
$$


Introducing the parameter $\lambda=\sqrt{W_{\mathrm{b}} c_{\mathrm{b}} / k}$, Eq. (28) can be rewritten as:

$$
\begin{aligned}
T= & T_{\mathrm{a}}+\frac{q_{0}}{2 k \lambda}\left[\mathrm{e}^{-\lambda x} \operatorname{erfc}\left(\frac{x}{\sqrt{4 \alpha t}}-\lambda \sqrt{\alpha t}\right)-\mathrm{e}^{\lambda x} \operatorname{erfc}\right. \\
& \left.\times\left(\frac{x}{\sqrt{4 \alpha t}}+\lambda \sqrt{\alpha t}\right)\right]
\end{aligned}
$$

Special case III: $W_{\mathrm{b}} c_{\mathrm{b}}=0$ and $q(0, t)=q_{0}$ The solution of this case can be easily obtained as:

$$
T=T_{\mathrm{a}}+2 \frac{q_{0}}{k} \sqrt{\alpha t} \operatorname{ierfc}\left(\frac{x}{\sqrt{4 \alpha t}}\right)
$$

For numerical stimulations, this study employed the thermal properties of tissue and blood based on the previous research [10]. The density of tissue is $1050 \mathrm{~kg} / \mathrm{m}^{3}$, both specific heat capacity of tissue and blood are $3770 \mathrm{~J} / \mathrm{kg} /{ }^{\circ} \mathrm{C}$, and the thermal conductivity of tissue is $0.5 \mathrm{~W} / \mathrm{m} /{ }^{\circ} \mathrm{C}$. In addition, the arterial temperature is $37^{\circ} \mathrm{C}$ and the heat flux on the skin surface ranges from 1000 to $5000 \mathrm{~W} / \mathrm{m}^{2}$ in this numerical study.

\section{Results and discussion}

When the time is zero, Fig. 1 shows the initial temperature profiles between Liu and $\mathrm{Xu}$ [13] and this study. In this figure, the dashed line represents the results of this study. And it is straight indicating the temperature maintains at $37^{\circ} \mathrm{C}$, which satisfies the initial condition. On the contrary, the continuous line represents the results of [13], and it is not constant through different depths. Because they assumed the temperature response was also steady periodic oscillation in order to match the boundary condition of sinusoidal heat flux. It is clear that the maximum difference between these two solutions occurs at the skin surface shown in Fig. 1. With the

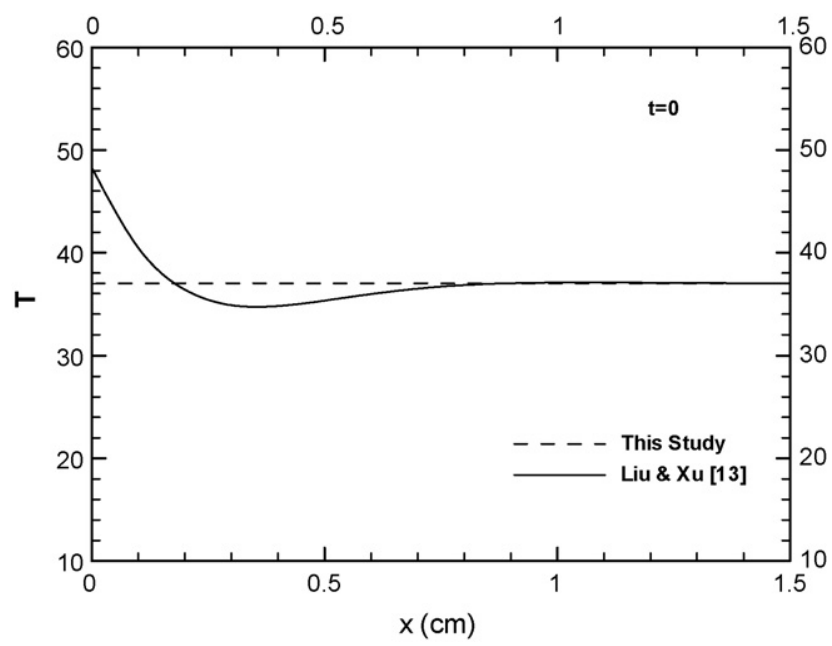

Fig. 1. Comparison between the solution of Liu and Xu [13] and Eq. (15) in this study on the temperature profiles along the distance from the surface skin when $t=0, \omega=0.05, W_{\mathrm{b}}=0.5 \mathrm{~kg} / \mathrm{m}^{3} / \mathrm{s}$, and $q_{0}=5000 \mathrm{~W} / \mathrm{m}^{2}$. increasing of the distance from the heating surface, the initial temperature difference between these two lines becomes smaller.

Fig. 2 shows the temperature response on the skin subjected to a sinusoidal heat flux. When the heat frequency on
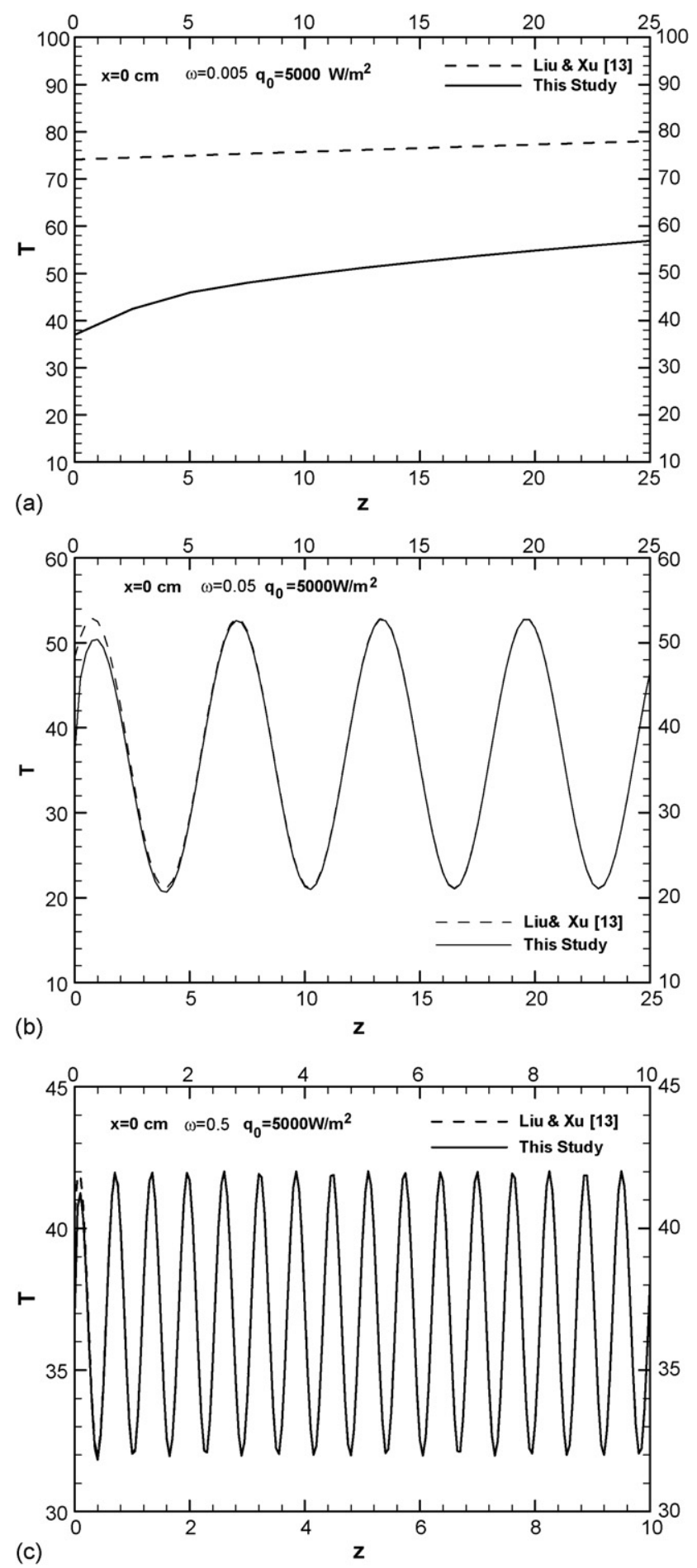

Fig. 2. Comparison of the transient temperature responses on the skin surface between Liu and Xu's solution [13] and Eq. (15) in this study at $q_{0}=5000 \mathrm{~W} / \mathrm{m}^{2}, W_{\mathrm{b}}=0.5 \mathrm{~kg} / \mathrm{m}^{3} / \mathrm{s}$, and (a) $\omega=0.005$; (b) $\omega=0.05$; (c) $\omega=0.5$. 
the skin surface is higher, the amplitude of temperature is lower. And the response cyclic time is longer when the heating frequency is lower. In the first cyclic period, the Liu and Xu's solution [13] is different from that of this study. Furthermore, the difference between the Liu and Xu's solution [13] and the exact solution of this study only occurs during the first cyclic period, especially for the lower heating frequency. Following the first cyclic period, the temperature deviation between the Liu and Xu's solution [13] and that of this study became smaller with increasing time. This implies that it still needs a little time to arrive at a steady periodic temperature response even for the steady periodic heat flux on the skin surface. In addition, Liu and Xu's solution [13] is the same with the present study for the final steady periodic oscillation of temperature response. On the other hand, the exact solution of this study can provide an appropriate way for describing the transient temperature response, especially in the early time of heating and for the lower heating frequency. Consequently, the analytical solution of this study can accurately predict the temperature response from the starting unsteady oscillation to the final steady periodic temperature oscillation.

In the initial period from $z=0$ to $z=8$ which has an equivalent time interval from 0 to $160 \mathrm{~s}$ when $\omega=0.05$ and $q_{0}=5000 \mathrm{~W} / \mathrm{m}^{2}$, Fig. 3 depicts the dimensionless temperature response and the dimensionless heat flux variation on the skin surface when $c_{1}=0.09524$. The continuous line represents the heat flux, as well as the dashed line and dash-dotted lines represent the results of this study and of Liu and Xu [13], respectively. It is clear that both peak temperatures and peak positions of the dashed line and dash-dotted line are different in the first cyclic period. As mentioned in the introduction, a noninvasive blood perfusion rate measurement is based on measuring the phase shift between the heat flux and temperature response at the skin. This method can estimate the blood perfusion rate after measuring the temperature response and getting the phase shift related to the heat flux phase angle on the skin surface. Because Liu and Xu's solution [13] is based

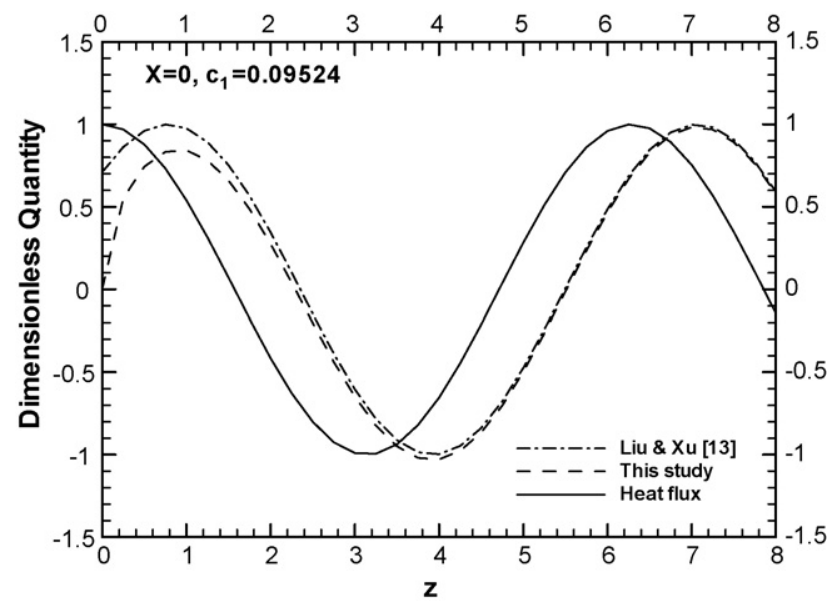

Fig. 3. Transient dimensionless temperature and heat flux on the skin surface when $c_{1}=0.09524$. on an assumption of steady periodic temperature oscillation over the whole time domain, the phase shift between heat flux and temperature are identical for each peak shown in Fig. 3. Nevertheless, Fig. 3 obviously shows that the phase shift between the heat flux and dashed line is different from that between heat flux and dash-dotted line in the first cyclic period. This indicates that the measurement of the phase shift in the first cyclic period cannot be used to estimate the perfusion rate of blood because of its intrinsic unstable character. The second peak of temperature response is close to phase angle of seven, and its equivalent time is about $140 \mathrm{~s}$ when the frequency is 0.05 . This implies that the beginning of sampling should be over $2 \mathrm{~min}$ in this case. Because the time to begin sampling phase shift increases with a decreasing frequency, it needs $10 \mathrm{~min}$ for waiting to sample the phase shift when the frequency of sinusoidal heat flux drops to 0.01 .

Fig. 4 demonstrates the dimensionless quantities of heat flux and temperature on the skin surface with different dimensionless blood perfusion rates when $\omega=0.05$ and $q_{0}=5000 \mathrm{~W} / \mathrm{m}^{2}$. In this figure, the long-dashed line and dashed line represents the temperature oscillation of $c_{1}=0.01$ and $c_{1}=0.1$, respectively. It is clear that both lines coincide with each other, and the difference of phase angle between the two lines is hard to distinguish. This means that it requires a highly precise device to detect the phase shift between the cases of $c_{1}=0.01$ and $c_{1}=0.1$. Moreover, the dash-dotted line and dash-double-dotted line represent the temperature oscillation of $c_{1}=1$ and $c_{1}=10$, respectively. Because larger amounts of blood perfusion rate can carry away more heat in a short time, the oscillatory amplitude decreases with the increasing of $c_{1}$. In addition, the phase shift between the temperature and sinusoidal heat flux oscillation becomes shorter with the increasing of the dimensionless blood perfusion rate. Therefore, detecting the variation of phase shift due to different blood perfusion rates is the principle of perfusion rate measurement. Referring to the Chato's handbook [21], the blood perfusion rate in human body is below $2.86 \mathrm{~kg} / \mathrm{m}^{3} / \mathrm{s}$.

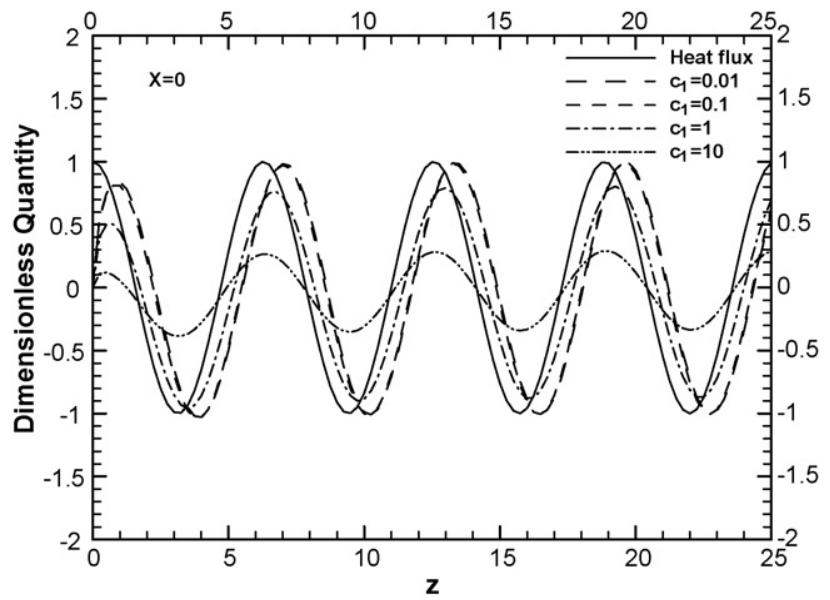

Fig. 4. Dimensionless temperature response and heat flux on the skin surface with different values of $c_{1}$. 


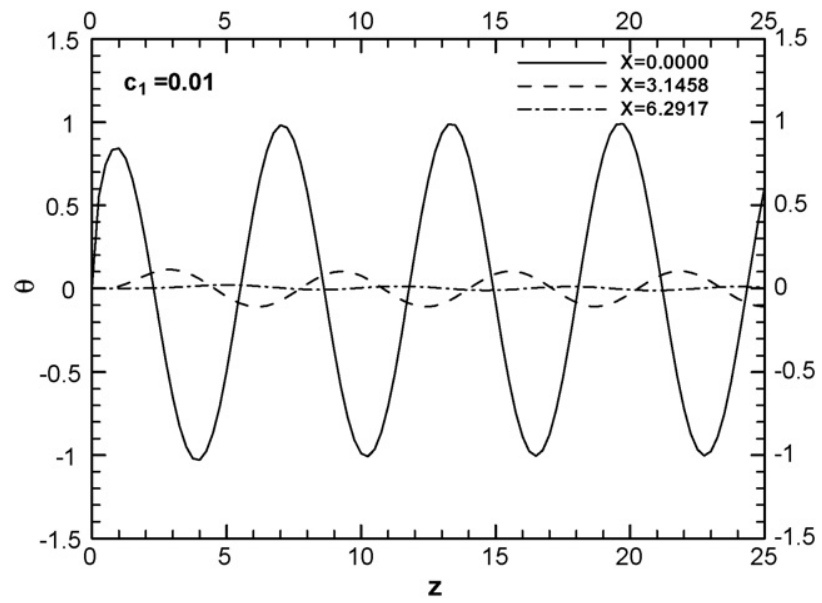

Fig. 5. Dimensionless temperature responses in different distances beneath the skin surface.

And the dimensionless blood perfusion rates of 0.01 and 0.1 in Fig. 4 are appropriate since they have equivalent blood perfusion rates of $0.525-5.525 \mathrm{~kg} / \mathrm{m}^{3} / \mathrm{s}$ when the frequency of sinusoidal heat flux is 0.05 . With the decreasing of frequency of sinusoidal heat flux, the dimensionless blood perfusion rate becomes larger. When the frequency of sinusoidal heat flux decreases to 0.01 , for example, the dimensionless perfusion rate is from 0.01 to 1 regarding the corresponding perfusion rate from 0.105 to $10.5 \mathrm{~kg} / \mathrm{m}^{3} / \mathrm{s}$. Therefore, the range of $c_{1}$ in Fig. 4 covers the application in medicine. When the blood perfusion rate remains unchanged, the dimensionless blood perfusion rate $c_{1}$ increases with the decreasing of the frequency. In this figure, all phase shifts in the first cyclic period are unavailable because all of them are still have unstable oscillation, which is mentioned in Fig. 3. The available time for beginning to sample is from 2 to $10 \mathrm{~min}$ when the frequency of sinusoidal heat flux decreases from 0.05 to 0.01 . Therefore, the beginning of the sampling needs more time to reach its final steady periodic oscillation of temperature response at the skin.

Fig. 5 depicts the dimensionless temperature responses at three different depths beneath the heating skin when $\omega=0.05$ and $q_{0}=5000 \mathrm{~W} / \mathrm{m}^{2}$. It is clear that the oscillatory amplitude of dimensionless temperature becomes to low for a larger depth from the heating skin. In this figure, the amplitude of temperature at $X=3.1458$ is about one-seventh of that at the skin. For example, the amplitude at $X=3.1458$ in Fig. 5 decreases around $4{ }^{\circ} \mathrm{C}$. This means that the tissue temperature at $5 \mathrm{~mm}$ beneath the skin oscillates between 35 and $39^{\circ} \mathrm{C}$. It is clear that the amplitude of the dimensionless temperature becomes very small while the distance beneath the heating skin is larger than $X=6.2917$. An examination of the dimensional temperature oscillation indicates that the amplitude is below $1^{\circ} \mathrm{C}$ when $X=6.2917$, which is at $1 \mathrm{~cm}$ beneath the skin. In addition, note that the frequency of the heating flux directly affects the depth with temperature oscillation, because the lower frequency has a better penetrative potential.

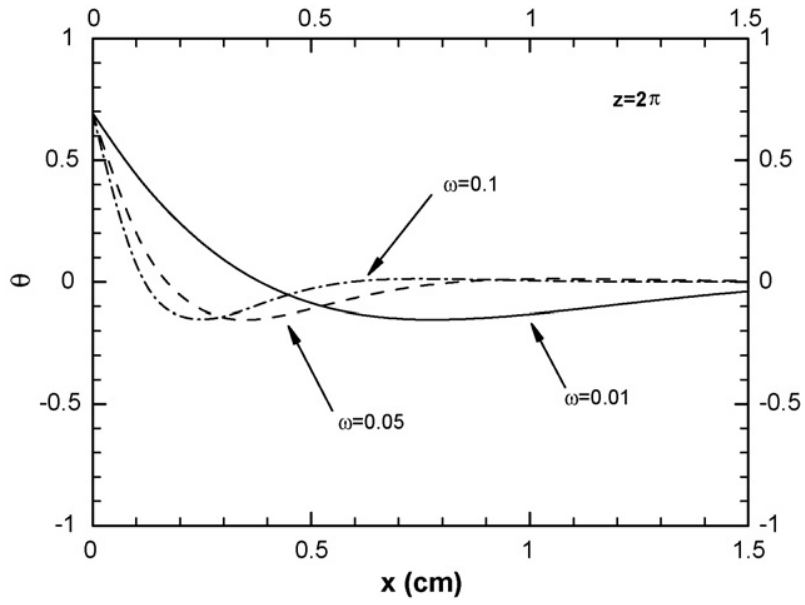

Fig. 6. Dimensionless temperature profile along the tissue depth at different frequencies of sinusoidal heat flux on the skin surface.

Fig. 6 depicts the temperature profile along the depth from the skin at different frequencies of sinusoidal heat flux proposed on the skin when $z=2 \pi, W_{\mathrm{b}}=0.5 \mathrm{~kg} / \mathrm{m}^{3} / \mathrm{s}$, and $q_{0}=5000 \mathrm{~W} / \mathrm{m}^{2}$. Meanwhile, the continuous, dashed, and dash-dotted lines represent the frequency of $0.01,0.05$, and 0.1 , respectively. It is clear that the depth influenced by the oscillation heat flux on the skin surface is below $1 \mathrm{~cm}$ when the frequency is higher than 0.05 . When the frequency decreases to 0.01 , the penetration depth exceeds $1.5 \mathrm{~cm}$.

Fig. 7 illustrates the dimensionless temperature profiles along the dimensionless distance changed with the different dimensionless times. Clearly, the temperature profile is substantially affected by the heating boundary, especially near $X=0$. When the dimensionless depth is larger than six, there is only a small oscillation of temperature. Furthermore, the temperature response after $X=6$ seems to be independent of the oscillatory heat flux on the heating skin. On the other hand, the effect of sinusoidal boundary heat flux on the tissue temperature is profound when $X$ is below six. According

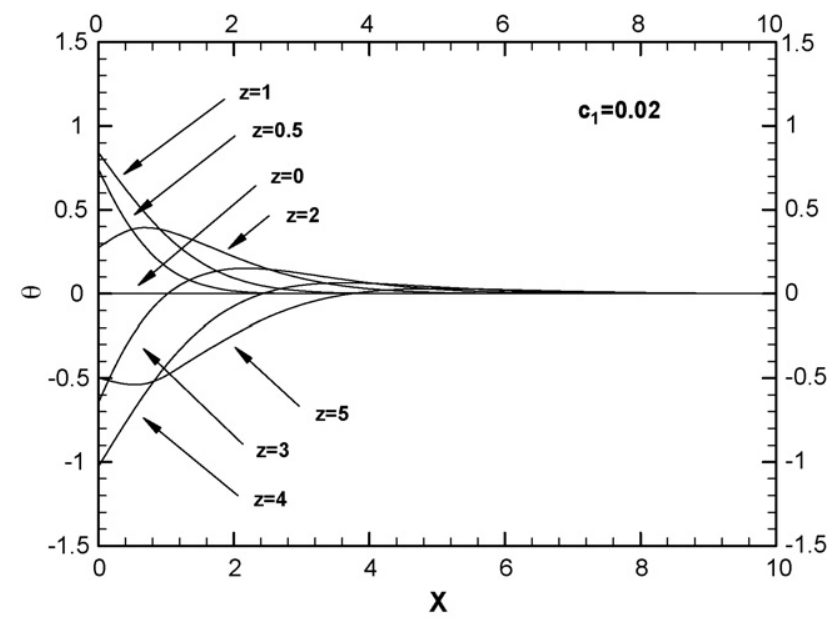

Fig. 7. Dimensionless temperature profiles along the dimensionless distance at different phase angles. 


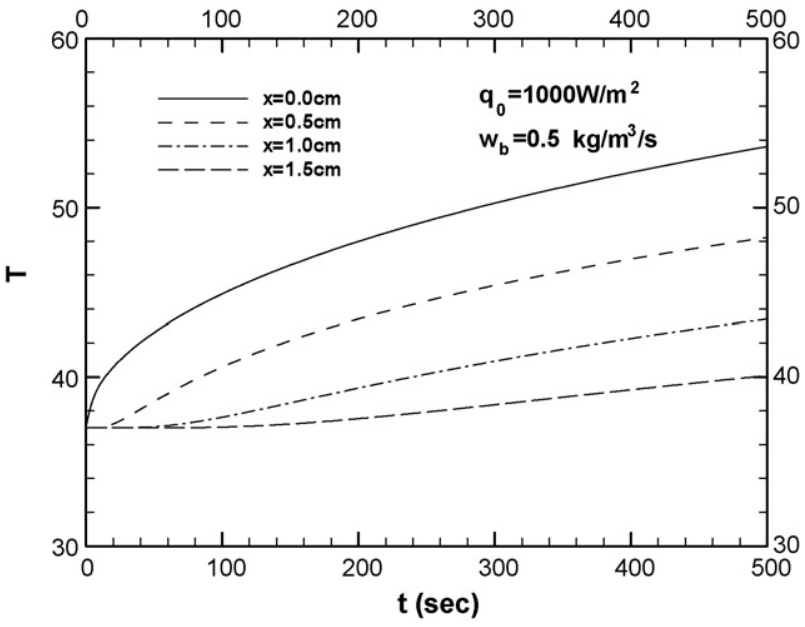

Fig. 8. Temperature response at different depths beneath the skin due to a constant heat flux of $1000 \mathrm{~W} / \mathrm{m}^{2}$.

to the definition of $X$, it is a function of position, thermal diffusivity, and frequency. Related to the frequency of 0.01 , 0.05 , and 0.1 , respectively, the affected depth will be close to 2,1 , and $0.7 \mathrm{~cm}$ when the thermal diffusivity is unchanged.

For the constant heat flux prescribed on the skin, Fig. 8 shows the influence on the temperature profiles with different distances from the skin. In this figure, the continuous, dashed, dash-dotted, and long-dashed lines represent the depth of 0 , $0.5,1.0$, and $1.5 \mathrm{~cm}$ beneath the skin, respectively. Due to the constant heat flux on the boundary, the temperature at different locations continuously increases over time. Meanwhile, the slope of lines decreases with the increasing of depth. This implies that the heating rate to the tissue becomes lower when the location is far from the heating surface. Therefore, the temperature arrives at $54{ }^{\circ} \mathrm{C}$ on the skin, meanwhile the temperature only rises to $40^{\circ} \mathrm{C}$ at $x=1.5 \mathrm{~cm}$ when the time is at $500 \mathrm{~s}$. Because the device of sinusoidal heat flux must alternately supply heating and cooling on the skin, it is difficult to apply in practice. Therefore, the reasonable boundary condition of blood perfusion rate measurement adds a constant heat flux into the Eq. (2) to avoid the occurrence of the negative heat flux on the skin. This study has derived the analytical expressions for the boundary conditions of sinusoidal heat flux and constant heat flux, respectively. Consequently, it is easy to predict the temperature response with boundary conditions of $q_{0}+q_{0} \mathrm{e}^{\mathrm{i} \omega t}$ by just adopting the mathematical superposition technique to combine the solutions (15) and (28).

\section{Conclusions}

This study investigates the effect of sinusoidal heat flux at the skin surface on the transient temperature field in a one-dimensional biological tissue. The exact solution of the Pennes bioheat transfer equation with the periodic heat flux boundary heating condition on the skin surface was derived in this work via the Laplace transforms. The analytical expressions obtained in this study are useful for describing the tissue temperature response in a one-dimensional domain, and especially it is more accurate than the results of the previous research in the initial period of time domain. According to the results, this study would like to draw some conclusions: (1) the first cyclic period of tissue temperature due to the sinusoidal heat flux boundary condition is still in a starting unstable oscillation. This means that the phase angle in this cyclic period is different from those in the final stable cyclic periods. Because the measurement of blood perfusion rate uses the phase shift between the temperature and heat flux oscillation wave to estimate the value of perfusion rate of blood, the phase shift in the first cyclic period of tissue temperature is unavailable. (2) The unavailable time of first oscillation for the estimation of the blood perfusion rate depends on the frequency of sinusoidal heat flux. It increases with the decreasing of the frequency. (3) Although it requires more time to wait for steady oscillatory temperature in lower frequency, the low frequency is still preferable for the measurement of blood perfusion rate because it can induce more obvious change of phase angle response to a blood perfusion rate difference.

\section{References}

[1] Liu J. Uncertainty analysis for temperature prediction of biological bodies subject to randomly spatial heating. J Biomech 2001;34:1637-42.

[2] Deng ZS, Liu J. Mathematical modeling of temperature mapping over skin surface and its implementation in thermal disease diagnostics. Comput Biol Med 2004;34:495-521.

[3] Chopra R, Wachsmuth J, Burtnyk M, Haider MA, Bronskill MJ. Analysis of factors important for transurethral ultrasound prostate heating using MR temperature feedback. Phys Med Biol 2006;51:82744.

[4] Jiang SC, Ma N, Li HJ, Zhang XX. Effects of thermal properties and geometrical dimensions on skin burn injuries. Burns 2002;28:713-7.

[5] Pennes HH. Analysis of tissue and arterial blood temperature in the resting forearm. J Appl Physiol 1948;1:93-122.

[6] Ng EYK, Chua LT. Comparison of one-and two-dimensional programmes for predicting the state of skin burns. Burns 2002;28:27-34.

[7] Chen C, Xu LX. Tissue temperature oscillations in an isolated pig kidney during surface heating. Ann Biomed Eng 2002;30:1162-71.

[8] Liu EH, Saidel GM, Harasaki H. Model analysis of tissue responses to transient and chronic heating. Ann Biomed Eng 2003;31:1007-48.

[9] Shih TC, Kou HS, Lin WL. The impact of thermally significant blood vessels in perfused tumor tissue on thermal dose distributions during thermal therapies. Int Commun Heat Mass Transfer 2003;30:975-85.

[10] Kou HS, Shih TC, Lin WL. Effect of the directional blood flow on thermal dose distribution during thermal therapy: an application of a Green's function based on the porous model. Phys Med Biol 2003;48:1577-89.

[11] Ocheltree KB, Frizzell LA. Determination of power deposition patterns for localized hyperthermia: a steady-state analysis. Int J Hyperthermia 1987;3:269-79.

[12] Ocheltree KB, Frizzell LA. Determination of power deposition patterns for localized hyperthermia: a transient analysis. Int J Hyperthermia 1987;4:281-96.

[13] Liu J, Xu LX. Estimation of blood perfusion using phase shift in temperature response to sinusoidal heating the skin surface. IEEE Trans Biomed Eng 1999;46:1037-43. 
[14] Deng ZS, Liu J. Analytical study on bioheat transfer problems with spatial or transient heating on skin surface or inside biological bodies. ASME J Biomech Eng 2002;120:638-49.

[15] Ng EYK, Sudharsan NM. An improved three-dimensional direct numerical modeling and thermal analysis of a female breast with tumour. Proc Inst Mech Eng Part H-J Eng Med 2001;215:25-37.

[16] Ng EYK, Sudharsan NM. Numerical uncertainty and perfusion induced instability in bioheat equation: its importance in thermographic interpretation. J Med Eng Tech 2001;25:222-9.

[17] Alekseev SI, Ziskin MC. Local heating of human skin by millimeter waves: a kinetics study. Bioelectromagnetics 2003;21:571-81.
[18] Alekseev SI, Radzievsky AA, Szabo I, Ziskin MC. Local heating of human skin by millimeter waves: effect of blood flow. Bioelectromagnetics 2005;26:489-501.

[19] Walters TJ, Blick DW, Johnson LR, Adair ER, Foster KR. Heating and pain sensation produced in human skin by millimeter waves: comparison to a simple thermal model. Health Phys 2000;78:259-67.

[20] Walters TJ, Ryan KL, Nelson DA, Blick DW, Mason PA. Effects of blood flow on skin heating induced by millimeter wave irradiation in humans. Health Phys 2004;86:115-20.

[21] Chato JC. Thermal properties of tissues. In: Skalak R, Chien S, editors. Handbook of Bioengineering. New York: Mcgraw-Hill; 1987. 